\title{
Wideband Spectrum Sensing on Real-Time Signals at Sub-Nyquist Sampling Rates in Single and Cooperative Multiple Nodes
}

\author{
Zhijin Qin, Student Member, IEEE, Yue Gao, Senior Member, IEEE, Mark D. Plumbley, Fellow, IEEE \\ and Clive G. Parini, Member, IEEE
}

\begin{abstract}
This paper presents two new algorithms for wideband spectrum sensing at sub-Nyquist sampling rates, for both single nodes and cooperative multiple nodes. In single node spectrum sensing, a two-phase spectrum sensing algorithm based on compressive sensing is proposed to reduce the computational complexity and improve the robustness at secondary users (SUs). In the cooperative multiple nodes case, the signals received at SUs exhibit a sparsity property that yields a low-rank matrix of compressed measurements at the fusion center. This therefore leads to a two-phase cooperative spectrum sensing algorithm for cooperative multiple SUs based on low-rank matrix completion. In addition, the two proposed spectrum sensing algorithms are evaluated on the TV white space (TVWS), in which pioneering work aimed at enabling dynamic spectrum access into practice has been promoted by both the Federal Communications Commission and the UK Office of Communications. The proposed algorithms are tested on the real-time signals after been validated by the simulated signals in TVWS. The numerical results show that our proposed algorithms are more robust to channel noise and have lower computational complexity than the state-of-the-art algorithms.
\end{abstract}

Keywords: Compressive sensing, low-rank matrix completion, sparsity property, spectrum sensing, TV white space.

\section{INTRODUCTION}

W ITH the rapid development of wireless communications, the scarcity of spectrum resources becomes an urgent problem. However, as reported by the Federal Communications Commission (FCC) and the UK Office of Communications (Ofcom), a large percentage of spectrum resources are underutilized [1], [2]. Cognitive radio (CR) was proposed to fully utilize spectrum resources by allowing unlicensed usage of vacant spectrum [3]. In CR, spectrum sensing is the first step to detect spectrum holes that can be used by secondary users (SUs) without causing any interference to primary users (PUs).

In recent research, wideband spectrum sensing has attracted much attention. A direct approach for wideband spectrum sensing is to acquire the wideband signals with a highspeed analog-to-digital converter (ADC) and then use digital signal processing techniques to detect spectral opportunities. Quan et al. [4] proposed a multi-band joint detection algorithm

The authors Zhijin Qin, Yue Gao and Clive G. Parini are with Queen Mary University of London, the School of Electronic Engineering and Computer Science, London E1 4NS, United Kingdom. (email:\{z.qin; yue.gao; c.g.parini\}@qmul.ac.uk)

The author Mark D. Plumbley is with the Centre for Vision Speech and Signal Processing (CVSSP), University of Surrey, Guildford, Surrey GU2 7XH, United Kingdom. (email:m.plumbley@surrey.ac.uk) to sense PUs over wideband spectrum by using a high-speed ADC for signal acquisition. Furthermore, Tian and Giannakis [5] proposed a wavelet-based wideband spectrum sensing algorithm by implementing a high-speed ADC. The wellknown Nyquist sampling theory requires that sampling rate should be at least twice of the signal bandwidth. Therefore, the sampling rate is very challenging for wideband spectrum sensing and the high-speed ADC for wideband signals is difficult or expensive to implement at SUs, especially for power-limited devices. Subsequently, Landau [6] demonstrated that sampling rate should be no less than the measure of the occupied part of the spectrum to guarantee the stable reconstruction of multiband signals which is below the corresponding Nyquist sampling rate. Recently, compressive sensing (CS) has been proposed to achieve sub-Nyquist sampling [7]. As the spectrum of interest is normally underutilized in reality [1], [2], the spectrum exhibits a sparse property in the frequency domain, which makes sub-Nyquist sampling possible by implementing the CS technique for spectrum sensing at SUs.

Tian and Giannakis [8] firstly introduced CS to wideband spectrum sensing, where fewer compressed measurements are required on the basis of Nyquist sampling theory. Subsequently, the wideband spectrum sensing based on CS has attracted much attention. In order to improve robustness against noise uncertainty, Tian et al. [9] proposed a cyclic detector by utilizing the CS principle for wideband spectrum sensing. In this algorithm, prior information of PUs such as sparsity level is considered to be known. However, the sparsity level is dependent on the spectrum occupancy which may be unavailable in dynamic CR networks. In order to solve this problem, Wang et al. [10] proposed a two-step CS scheme for minimizing the sampling rates when the sparsity level is changing. In this approach, the actual sparsity level is estimated firstly and the number of compressed measurements to be collected are then adjusted before taking samples. However, this algorithm introduces extra computational complexity by performing the sparsity level estimation. In order to remove the sparsity level estimation step, Sun et al. [11] proposed to adjust the number of compressed measurements adaptively by acquiring compressed measurements step by step in continuous sensing slots. However, this iterative process introduces higher computational complexities at the $\mathrm{SU}$ as signal reconstruction has to be performed several times until the exact signal recovery is achieved.

In addition, some research work on the implementation of compressive spectrum sensing for the continuous-time 
signal acquisition has been reported recently. For example, Venkataramani et al. [12] proposed the multi-coset sampling and it is utilized in [13]-[15] to reduce the sampling rate for the case of multiband signals. Similarly, Mishali and Eldar [16], [17] proposed a modulated wideband converter model for multichannel sub-Nyquist wideband sensing, and the minimal sampling rate for exact signal recovery has been derived by the authors. Tropp et al. [18] proposed an analogto-information converter (AIC) sampler to make the analog CS implementable, and the restricted isometry property has been proved enabling the exact signal recovery to be guaranteed with a cost of more measurements. In addition, the AIC sampler has been adopted in [10].

Furthermore, we noticed that most of the existing algorithms [19]-[21] normally do not specify or quantify the noise. The signal-to-noise ratio (SNR) of the CS measurements would be decreased by $3 \mathrm{~dB}$ for every octave increasing in the subsampling factor for acquisition of a noisy signal with fixed sparsity [22], which makes it more difficult for CS based spectrum sensing under heavy channel noise. Therefore, a robust spectrum sensing algorithm based on CS with low computational complexity is demanding.

In this paper, we propose a two-phase spectrum sensing algorithm based on CS for a single node scenario. In the first phase, a new wideband channel division scheme is proposed to reduce the computational complexity of the signal recovery process. In the second phase, a denoising algorithm is performed to improve detection performance and enable the algorithm to be more robust to channel noise. To this end, the proposed algorithm is validated by the simulated signals and tested on the recorded real-time TV white space (TVWS) signals obtained by the CRFS RFeye node [23].

Single node spectrum sensing faces the challenges that detection performance is significantly degraded if an SU experiences multipath fading and hidden terminals [24], [25]. This may cause miss detection. As a result, the SU may unwittingly transmit signals in channels with active PUs, which may cause serious interference to the PUs. In order to reduce the influence of imperfect channel environment, multiple nodes spectrum sensing, named as cooperative spectrum sensing (CSS), was proposed to efficiently combat fading problems by utilizing a spatial diversity of cooperative multiple SUs [4], [19], [26].

In CSS networks, there are two types of data fusion: centralized and decentralized fusion. In decentralized CSS, each SU only communicates with its neighbour SUs within one hop to reduce the transmission power consumed during sensing. After convergence, all SUs will have the fused sensing result without the implementation of a fusion center (FC). Several decentralized CSS schemes [27]-[29] have been proposed where the average value of all the local spectrum sensing decisions is computed to get the final decision. As a result, the final decision obtained might be sub-optimal. In addition, Zeng et al. [19] proposed a distributed CSS algorithm in which sensing samples rather than sensing decisions are exchanged with the neighbour SUs within multi-hops to reach a global fusion at the cost of increasing network load. The convergence speed of decentralized CSS is an issue in large scale networks. In the centralized CSS scheme, all SUs report to an FC to make a final decision. In existing algorithms [30], [31], each
SU makes a local decision about the spectrum of interest, and then the local decisions are sent to an FC to make a final decision. For a multi-channel sensing algorithm, such a separate approach of local spectrum estimation followed by a global decision fusion is suboptimal, which it does not take full advantage of the spatial diversity of the cooperative SUs [20]. In [19], [32], [33], an SU senses the whole spectrum of interest, and then the SU sends all the collected compressed measurements to an FC to get a global decision. As a result, the optimal decision can be obtained but the transmission load in the reporting channel between SUs and the FC is heavy. In [20], in order to reduce the sampling costs and transmission load between SUs and the FC, the length of received signal's frequency domain representations is set to be equal to the number of channels in the spectrum of interest rather than the original length of received signal in time domain, which results in a very poor resolution in the frequency domain and serious spectral leakage in each channel. Consequently, the false alarm probability $P_{f}$ and miss detection probability $P_{m}$ increase. In addition, as aforementioned, the noise would becomes heavier after signals are collected at sub-Nyquist rate [22]. Therefore, a sub-Nyquist sampling based CSS algorithm with high spectrum resolution, low computational complexity and high robustness to noise is required.

In this paper, a two-phase CSS algorithm based on lowrank matrix completion (MC) is proposed to reduce the signal acquisition costs at SUs and the spectrum resolution and improve the robustness to channel noise. In the first phase, the proposed wideband channel division scheme is implemented at each SU to reduce signal acquisition costs. The received samples are transformed into frequency domain with their original length to eliminate spectral leakage. Sequentially, only the compressed measurements are sent to an FC with reduced transmission load in the CSS network. At the FC, $\mathrm{MC}$ is performed on the incomplete matrix constructed by compressed measurements from SUs. In the second phase, detection performance is further improved by a denoising algorithm. In addition, the proposed algorithm is tested on the real-time signals after been validated by the simulated signals.

The rest of this paper is organized as follows. Section II describes the proposed two-phase spectrum sensing algorithm based on CS for the single node. Numerical analyses for the proposed algorithm is demonstrated in section III. Section IV develops the proposed two-phase CSS algorithm based on low-rank MC. Numerical results for the proposed two-phase CSS algorithm on both simulated and real-time signals are presented in section V. Conclusions are drawn in section VI.

\section{Proposed Two-Phase Single Node Spectrum} SEnsing Algorithm BAsed on Compressive SENSING

\section{A. System model}

A four-step spectrum sensing based on CS is summarized as follows:

1) Sparse signals received at SUs.

2) Compressed measurements collection at SUs.

3) Signal recovery at SUs.

4) Decision making at SUs. 
1) Sparse signals received at $S U s$ : It is assumed that bandwidth of the whole spectrum is $B \mathrm{~Hz}$. The signal received at an $\mathrm{SU}$ is given by:

$$
r(t)=h(t) * s(t)+w(t),
$$

where $s(t) \in C^{N \times 1}$ is the time domain representation of the transmitted signal, $h(t)$ is the channel gain between the transmitter and receiver, and $w(t) \sim \mathcal{C N}\left(0, \sigma^{2} \mathbf{I}_{N}\right)$ refers to additive white Gaussian noise (AWGN) where $\sigma^{2}$ refers to noise variance and $\mathbf{I}_{N}$ is the identity matrix. In order to simplify the system, we assume $h(t) \equiv 1$ until further specified.

In order to make sure the CS technique working at SUs to reduce the sampling rate, the received signal should be able to be expressed in a sparse domain. In spectrum sensing, the signals received at an SU $r(t)$ is assumed to be sparse in the frequency domain and it can be expressed as $r_{f}=s_{f}+w_{f}$, where $r_{f}, s_{f}$ and $w_{f}$ are the discrete Fourier transform (DFT) of $r(t), s(t)$ and $w(t)$. As aforementioned, $s_{f}$ is sparse since the spectrum is normally underutilized.

2) Compressed measurements collection at SUs: After CS technique is applied at an SU, the collected compressed measurements can be expressed as:

$$
x=\Phi \mathcal{F}^{-1} r_{f}=\Theta r_{f}=\Theta\left(s_{f}+w_{f}\right),
$$

where $\Phi \in C^{P \times N}(P \leq N)$ is a measurement matrix to collect the compressed measurements $x \in C^{P \times 1}$, with $P / N \leq 1$ being the compression ratio. The measurement matrix can be a matrix which contains a single spike in each row. The case $P / N=1$ corresponds to $\Phi=\mathbf{I}_{N}$. In addition, $\Theta=\Phi \mathcal{F}^{-1}$, where $\mathcal{F}^{-1}$ is inverse DFT matrix which is used as the sparsifying matrix.

In practical settings, structured random matrices are often employed for improved implementation affordability. We adopt AIC sampler proposed in [18] to realize the sub-Nyquist sampling in our paper. The AIC sampler mainly contains three components: a high-rate pseudonoise sequence, a lowpass anti-alising filter and a low speed ADC. This well designed structure alleviates the burden on the ADC, at the expense of slightly degraded recovery performance compared with those fully random Gaussian sampler. There are three conditions for the measurement matrix: i) each column of it is normalized, ii) each row of it has approximately equal norm, and iii) the rows of it are orthogonal. These three conditions can be fulfilled by random matrices such as AIC sampler [22].

3) Signal recovery at SUs: When the CS technique is implemented at SUs, sampling rates are reduced to sub-Nyquist sampling rates. However, in order to make accurate decisions about spectrum occupancy, the signal recovery should be performed by solving the following $l_{1}$ norm minimization problem:

$$
\hat{s}_{f}=\arg \min \left\|\hat{s}_{f}\right\| \text {, s.t. }\left\|\Theta \cdot \hat{s}_{f}-x\right\|_{2}^{2} \leq \varepsilon .
$$

where $\varepsilon$ is the noise tolerance.

4) Decision making at SUs: When the estimated signal $\hat{s}_{f}$ is obtained, energy detection is performed to determine the spectrum occupancy. Specifically, the energy density of each channel in the recovered signal is compared with a predefined threshold to make a decision. The predefined threshold $\lambda_{d}$ is dependent on the noise variance $\left(\sigma^{2}\right)$, the target $\mathrm{P}_{f}$ and the number of samples $(N)$ [34]:

$$
\lambda_{d}=\sigma^{2}\left(1+\frac{Q^{-1}\left(P_{f}\right)}{\sqrt{N / 2}}\right),
$$

where $N$ is the sample number of the original signal. In practice, the noise power $\left(\sigma^{2}\right)$ can be calibrated in a given channel which is known for sure to be idle. For example, some channels such as channel 21 in TVWS are supposed to be vacant currently in the UK.

If the energy density of each considered channel is higher than the threshold, the corresponding channel is determined as occupied by PUs, and SUs are forbidden to access it. Otherwise, the corresponding channel is determined as vacant, and SUs can access it to transmit unlicensed signals.

\section{B. The proposed two-phase single node spectrum sensing algorithm based on compressive sensing}

As we do not require any prior information about the PUs, $l_{1}$ norm minimization is adopted to perform the signal recovery. In order to reduce the computational complexity during signal recovery process and enhance algorithm's robustness to imperfect channel noise, we propose a two-phase spectrum sensing algorithm for single node based on CS. In the first phase, a new efficient channel division scheme is proposed to reduce the computation complexity for signal recovery. In the second phase, a denoising algorithm is proposed to enable the algorithm to be more robust against heavy channel noise.

1) The efficient channel division scheme: When an $l_{1}$ norm minimization based spectrum sensing algorithm is implemented at an SU, the computational complexity of signal recovery is dependent on the number of samples to be recovered. It is assumed that there are $L$ channels in the spectrum of interest. We propose a new channel division scheme in which only $K(K<L)$ out of $L$ channels are expected to be sensed in one sensing period at SUs to reduce the number of samples to be recovered. As shown in Fig. 1, each $K$-channel group is indexed by $i\left(i=1,2, \cdots, \frac{L}{K}\right)$. If any vacant channel is detected, SU would stop sensing and start data transmission. Otherwise, SU begins to sense the next $K$-channel group in the next sensing period. As a result, the required sampling rates at SUs for exact recovery are further reduced by implementing CS technique at SUs.

Once signal of the $K$-channel group $s_{f i} \in C^{n \times 1}(n=$ $\frac{K N}{L}$ ) arrives at the receiver, compressed measurements $x_{i}$ are collected at sub-Nyquist sampling rates. Subsequently, the recovered frequency domain representations of the $i$ th $K$-channel group $\hat{s}_{f i}$ can be obtained by solving $l_{1}$ norm minimization as:

$$
\hat{s}_{f i}=\arg \min \left\|\hat{s}_{f i}\right\|_{1} \text {, s.t. }\left\|\Theta_{i} \cdot \hat{s}_{f i}-x_{i}\right\|_{2}^{2} \leq \varepsilon_{i},
$$

where $\Theta_{i} \in C^{p \times n}, p=\frac{K P}{L}$ and $\varepsilon_{i}$ is the error tolerance in the reconstruction process for signal $\hat{s}_{f i}$.

2) The denoised spectrum sensing algorithm: When making a decision for spectrum occupancy, the decision accuracy is influenced by the signal recovery errors. The recovery performance of traditional $l_{1}$ norm minimization algorithm is degraded by heavy channel noise and low compression 


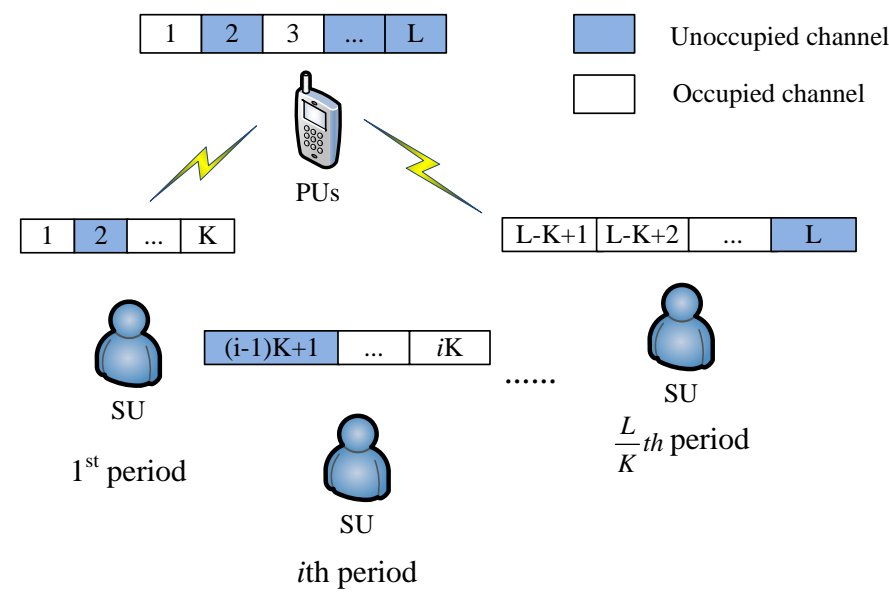

Fig. 1: System model of the proposed channel division scheme in the single node spectrum sensing based on compressive sensing.

ratio. Furthermore, it is noticed that the amplitudes of recovered signal $\hat{s}_{f i}$ may be negative with high absolute values. However, the power spectrum $s_{f i}$ is nonnegative. If those negative values are used to calculate the energy density, it would become higher than the real energy value. As a result, the $P_{f}$ of spectrum sensing would increase, which means the vacant channels might be determined as occupied. In order to improve the recovery performance and detection performance, a denoising algorithm is proposed.

In the denoising algorithm, the amplitude of the $b$ th frequency bin in the recovered signal $\hat{s}_{f i}$ is compared with the corresponding noise level $\sigma(b)$, where $b$ is the index of the recovered signal. If $\hat{s}_{f i}(b)$ is higher than $\sigma(b)$, the compressed measurement collected at SUs $r_{f i}(b)$ is kept for the recovered signal. Otherwise, the corresponding value will be set to zero to reduce the recovery error. The denoised signal $\hat{s}_{f i_{-} d}$ can be expressed as:

$$
\hat{s}_{f i \_d}(b)=\left\{\begin{array}{cc}
r_{f i}(b) & \text { if } \hat{s}_{f i}(b) \geq \sigma(b) \\
0 & \text { otherwise }
\end{array} .\right.
$$

After the denoising algorithm is performed, the energy density of each considered $K$-channel group in the denoised signal is compared with the corresponding threshold as defined in (4) to determine the spectrum occupancy of the corresponding $K$-channel group. If any $K$-channel group are determined as vacant, they can be used by SUs to transmit the unlicensed signals. Otherwise, the SU should continue sensing the next $K$-channel group until any vacant channel is found out or the $\frac{L}{K}$ sensing periods, named as a sensing loop, are run out. As there is a high probability that the spectrum vacant in last loop remains free in the current sensing loop, an SU should firstly sense the $K$-channel group determined as free in the last sensing loop at the beginning of a new sensing loop if any vacant $K$-channel group are detected in the most recent sensing loop. Otherwise, an SU should keep sensing from the first $K$-channel group. The whole process of the proposed twophase spectrum sensing algorithm at single node based on CS is summarized as Algorithm 1.
Algorithm 1 Two-phase Single Node Spectrum Sensing Algorithm based on Compressive Sensing

\section{Initialization:}

Set threshold $\lambda_{d}$ as (4), $i=1$.

1: while $i \leq \frac{L}{K}$ or $E\left(\hat{s}_{f i} d\right)<\lambda_{d}$ do

\section{Phase I:}

2: The SU takes measurements at sub-Nyquist rate for the $i$ th $K$-channel group to collect $r_{i}$ in the $i$ th sensing period.

3: Perform signal recovery by $l_{1}$ algorithm as (5) to get the recovered signal $\hat{s}_{f i}$.

\section{Phase II:}

4: $\quad$ Perform denoising to $\hat{s}_{f i}$ to get $\hat{s}_{f i_{-} d}$.

5: $\quad$ Increase $i$ by 1 .

6: end while

\section{Decision:}

If $E\left(\hat{s}_{f i \_}\right)<\lambda_{d}$, SU can access the $i$ th $K$-channel group. An SU senses from the $K$-channel group vacant in last sensing loop or from the first $K$-channel group in the new sensing loop.

C. Computational complexity and spectrum efficiency analyses

In compressive spectrum sensing algorithm, the computational complexity mainly comes from the signal recovery process by solving the $l_{1}$ norm minimization problem. It is determined by the number of samples $(N)$ to be recovered to represent the spectrum of interest. Specially, when the whole wideband spectrum of interest is sensed in one sensing period by an $\mathrm{SU}$, the computational complexity of solving the $l_{1}$ norm minimization problem can be expressed as:

$$
C_{1}=\mathrm{O}\left(N^{3}\right) .
$$

In the adaptive compressive spectrum sensing algorithm [11] for wideband CRs, the required computational complexity $C_{2}$ can be expressed as follows. In order to simplify the comparison, the spectrum sensed in each sensing period is assumed to be $K$ out of $L$ channels and the system starts data transmission after $i$ sensing periods.

$$
\begin{aligned}
C_{2} & =\mathrm{O}\left(\left(\frac{K}{L} \times n\right)^{3}+\left(\frac{2 K}{L} \times N\right)^{3}+\ldots+\left(\frac{i K}{L} \times N\right)^{3}\right) \\
& =\mathrm{O}\left(\left(1+2^{3}+\ldots+i^{3}\right) \times\left(\frac{K}{L}\right)^{3} \times N^{3}\right) \\
& =\mathrm{O}\left(\left(\frac{(1+i) i}{2}\right)^{2} \times\left(\frac{K}{L}\right)^{3} \times N^{3}\right),
\end{aligned}
$$

where $i=1, \ldots, \frac{L}{K}$ is the number of sensing periods that an $\mathrm{SU}$ needs to perform exact signal recovery to determine the accessible channels.

When the proposed new channel division scheme is used for single node wideband spectrum sensing, computational complexity of the signal recovery process is expressed as:

$$
C_{3}=\mathrm{O}\left(i \times\left(\frac{K}{L}\right)^{3} \times N^{3}\right) .
$$


As analysed above, an SU may need multiple sensing periods to find out the accessible spectrum holes. Assuming there is at least one vacant channel in the spectrum of interest, the proposed channel division scheme requires $\frac{K}{L}$ sensing periods, which is dependent on the number of channels in a $K$-channel group. The worst case for the proposed scheme is that an SU does not find any vacant channel until the $\frac{K}{L}$ th sensing period. In such a case, $C_{3}=\mathrm{O}\left(\left(\frac{K}{L}\right)^{2} \times N^{3}\right)$ with $i=\frac{L}{K}$. In practice, there are multiple vacant channels in the spectrum of interest due to the low spectrum utilization. Therefore, the required sensing periods would be less than $\frac{K}{L}$ in reality. As a result, $C_{1}>C_{3}$ in all cases. The proposed channel division scheme relaxes the requirement on high speed ADC at the expense of compromised spectrum efficiency. This tradeoff is shown in the simulation part and it seems acceptable as the proposed algorithm is designed for machine-to-machine (M2M) communications in which SUs have limited computational power and infrequent low-speed transmission requirements. Comparing $C_{2}$ and $C_{3}$, we can see that $C_{2}=C_{3}$ if $i=1$, which refers to the scenario that vacant channels can be found after signal recovery is only performed once. Otherwise, $C_{2}>C_{3}$. Therefore, the proposed channel division scheme achieves a lower computational complexity than existing algorithms.

\section{Numerical Analyses on Proposed Two-Phase Single Node Spectrum Sensing Algorithm Based ON COMPRESSIVE SENSING}

\section{A. Analyses on simulated signals}

In the simulation, signals are orthogonal frequency-division multiplexed (OFDM) generated as PUs, which are used in Digital Video Broadcasting-Terrestrial (DVB-T) over the TVWS spectrum from $470 \mathrm{MHz}$ to $790 \mathrm{MHz}$ in the UK [2]. There are $L=40$ channels in TVWS with bandwidth of $8 \mathrm{MHz}$ for each channel. $P_{f}$ is set to be 0.01. $S N R=\sigma_{s}^{2} / \sigma^{2}$ is the ratio of signal power over noise power of a $K$-channel group. In the following simulations, the aforementioned tradeoff between spectrum efficiency and computational complexity is demonstrated firstly. In addition, the influence of compression ratio, sparsity order and the classic receiver operating characteristics (ROC) curves are presented to validate the proposed algorithm.

Fig. 2 shows the average number of sensing periods $\bar{i}$ which is required at SUs to find out the vacant channel for unlicensed usage. As aforementioned, the size of $K$-channel group which is sensed in each sensing period at SUs would influence the spectrum efficiency of the proposed channel division scheme. If $K=1$, the case becomes a narrow band spectrum sensing which requires low-speed sampling rates at SUs. But the spectrum efficiency is low. With increasing $K$, it becomes a multichannel wideband spectrums sensing case in which the spectrum efficiency is increased with cost of expensive sampling acquisition. From Fig. 2, we can see that the vacant channels can be detected efficiently even with increasing $K$. With higher sparsity level which refers to higher spectrum occupancy, the average number of sensing periods increases. As the spectrum is underutilized in practice, the required number of sensing periods is relatively low. In the following simulation, it is assumed that the number of channels sensed by the $\mathrm{SU}$ in each sensing period is set to be $K=8$.

Fig. 3 shows detection probability $P_{d}$ for the traditional $l_{1}$ norm minimization based spectrum sensing algorithm and the proposed two-phase single node spectrum sensing algorithm based on CS under different number of compressed measurements with varying SNR values. Its detection performance is also compared with that of spectrum sensing algorithm without CS implemented, as well as the theoretical values derived from [35], [36]:

$$
P_{d}=Q\left(\frac{\frac{\lambda_{d}}{\sigma^{2}}-\left(1+\frac{\sigma_{s}^{2}}{\sigma^{2}}\right)}{\left(1+\frac{\sigma_{s}^{2}}{\sigma^{2}}\right) / \sqrt{N / 2}}\right),
$$

where $\lambda_{d}$ is the threshold for energy detection as calculated by (4), and $\sigma_{s}^{2}$ refers to signal power.

Fig. 3 shows that the performance of $l_{1}$ norm minimization based spectrum sensing algorithm (labeled as traditional CS based SS) and the proposed two-phase single node spectrum sensing algorithm based on CS (labeled as denoised CS based SS) are both the same with that of spectrum sensing algorithm without CS implemented at the SU (labeled as SS without CS) and the theoretical curves obtained by (10). In this case, the number of occupied channels is 1 among 8. Therefore, the sparsity level is set to be $12.5 \%$. When the number of collected measurements decreases, the detection performance degrades. It also shows that performance of the proposed two-phase single node spectrum sensing based on CS is better than that of the CS based spectrum sensing without denoising when the compression ratio is $25 \%$ and $10 \%$. This gain benefits from the proposed denoising algorithm which can improve the signal recovery accuracy. As the recovery accuracy becomes higher with the higher compression ratio, detection performance of the proposed two-phase spectrum sensing algorithm gets closer to the theoretical curves. The simulation result shows that the proposed two-phase spectrum sensing algorithm can reduce the sampling rates by $75 \%$ without degrading detection performance.

Fig. 4 shows the detection performance of the proposed two-phase two-phase single node spectrum sensing based on CS with different sparsity levels and different compression ratios. In this scenario, the different sparsity levels refer to different number of active PUs in the spectrum of interest. The positions of these active PUs are set to be random. The detection performance becomes worse with increasing sparsity level and decreasing compression ratio as shown in Fig. 4. As the sparsity level increases, sparse property of signal to be recovered becomes less sparse, and therefore more compressed measurements should be collected for signal recovery to make sure the detection performance not being degraded. It is noticed that the detection performance would only be slightly degraded when the proposed algorithm is applied to the practical signals in TVWS spectrum as the its occupancy ratio is normally $15 \%$ to $20 \%$ in practice [1], [2].

The ROC curves under different SNR values are shown in Fig. 5, where the compression ratio is set to be $25 \%$. In this case, the sparsity level is set to be $12.5 \%$. It can be observed that the proposed two-phase spectrum sensing algorithm based on CS exhibits better performance than the 


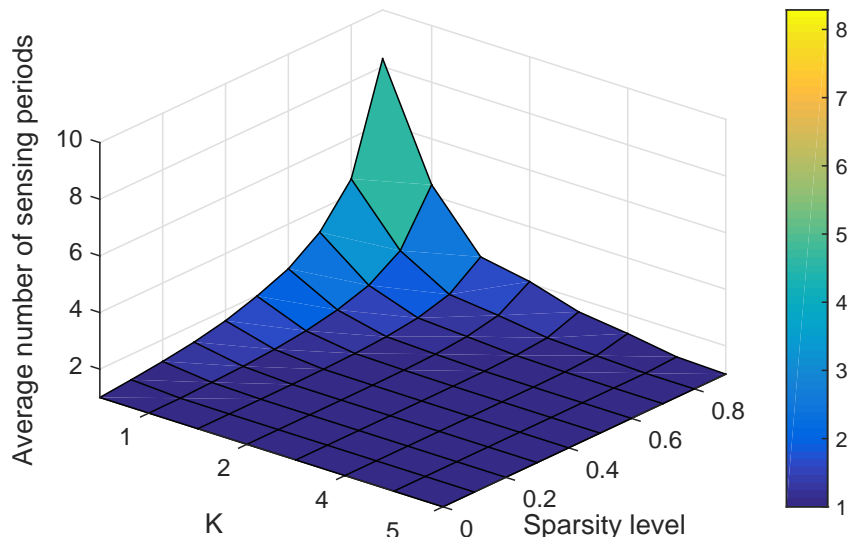

Fig. 2: Average number of required sensing periods at SUs with different sparsity levels and number of channels $K$ sensed in one sensing period.

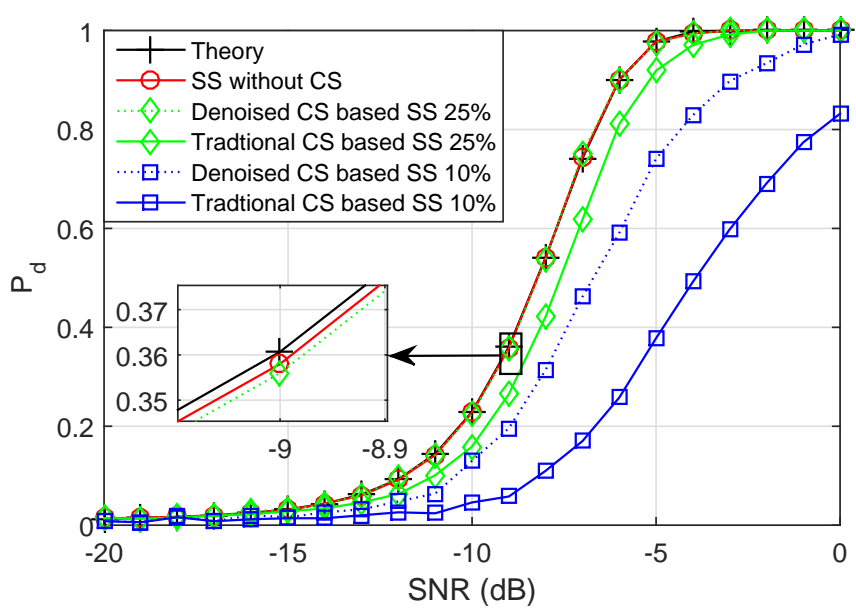

Fig. 3: Proposed denoised compressive spectrum sensing algorithm at single node achieves higher detection probability $P_{d}$ than the traditional algorithms with simulated signals under different compression ratios and different SNR values.

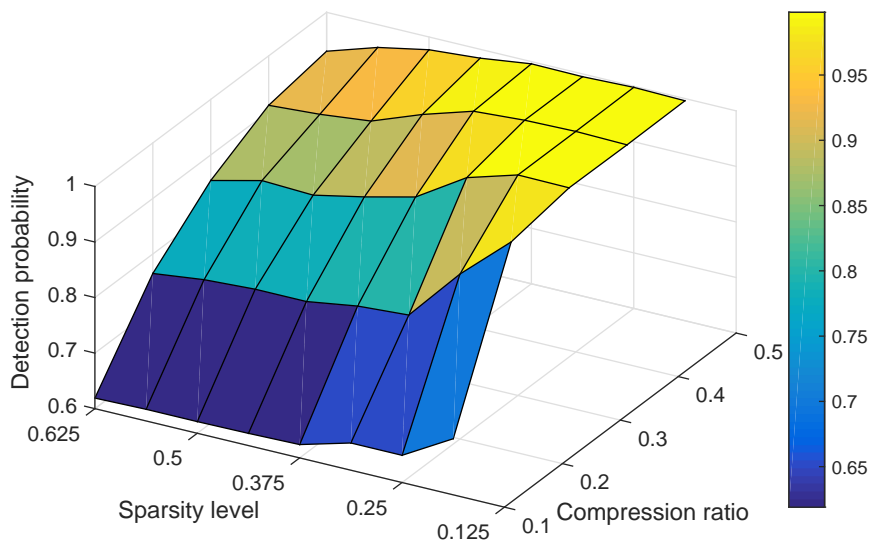

Fig. 4: Probability of detection $P_{d}$ comparison with different sparsity levels and different compression ratios. traditional spectrum sensing algorithm based on CS. Meanwhile, it is also noticed that the performance of the proposed two-phase spectrum sensing algorithm is almost as good as that of spectrum sensing algorithm without CS applied. This gain arises from the proposed denoising algorithm. This result matches with Fig. 3 when compression ratio is set to be $25 \%$. It should be pointed that the increasing $P_{f}$ refers to decreasing threshold level if the number of samples is fixed as defined in (4). Therefore, the detection performance becomes degraded with increasing threshold level as shown in Fig. 5. This work has been preliminarily demonstrated in our previous study [37], which gives us the confidence to test the proposed algorithm on the real-time signals in the following.

\section{B. Analyses on real-time signals}

After the proposed two-phase single node spectrum sensing algorithm has been validated with simulated signals, we test it on real-time signals recorded by the RFeye node. The RFeye node is a scalable and cost-effective node which can provide real-time $24 / 7$ monitoring of radio spectrum. It is capable of sweeping spectrum from $10 \mathrm{MHz}$ to $6 \mathrm{GHz}$, and can capture signals of all types, including transient transmission such as pulsing or short-burst signals. It is even sensitive to very low power signals. The RFeye node used for measurement is located at $\left(51.523021^{\circ} \mathrm{N} 0.041592^{\circ} \mathrm{W}\right)$ as shown in Fig. 6 with the height about 15 meters above ground. The real-time signal recorded by the RFeye node is for TVWS ranging from $470 \mathrm{MHz}$ to $790 \mathrm{MHz}$.

When the recorded real-time signal is used as source signal for the proposed two-phase single node spectrum sensing algorithm, Fig. 7 shows $P_{d}$ and $P_{f}$ of the spectrum sensing without CS implemented, traditional the proposed denoised spectrum sensing algorithms under different threshold values. Here, the thresholds are experimental values. In this scenario, the compression ratio is set to be $15 \%$. We can see that both $P_{d}$ and $P_{f}$ decrease with increasing threshold values. IEEE 802.22 demands a stringent sensing requirement. For the maximum $P_{f}$ of $10 \%$, a sensing algorithm should achieve $90 \%$ for $P_{d}$ [38]. According to the Fig. 7, we can see that the detection performance of the spectrum sensing without CS implemented can achieve the target performance required in IEEE 802.22 when threshold is set to be 1.5 or higher. However, the $P_{d}$ of the algorithms with CS would be degraded with increasing threshold. Therefore, we choose 1.5 as the suitable threshold to get a better tradeoff of $P_{d}$ and $P_{f}$ in the following analyses. From Fig. 7, it is also noticed that the proposed two-phase single node spectrum sensing algorithm outperforms the traditional one when threshold is 1.5 . We can see that the $P_{d}$ increases with decreasing threshold level which is matched with the simulation results shown in Fig. 5.

Fig. 8 shows the $P_{d}$ and $P_{f}$ of the traditional spectrum sensing algorithm based on CS and the proposed two-phase spectrum sensing algorithm with real-time signals under different compression ratios from $1 \%$ to $100 \%$. In this scenario, the threshold value is set to be 1.5 according to Fig. 7 . We can see that the detection performance gets better with increasing number of compressed measurements collected at the SU, and the proposed two-phase spectrum sensing algorithm outper- 


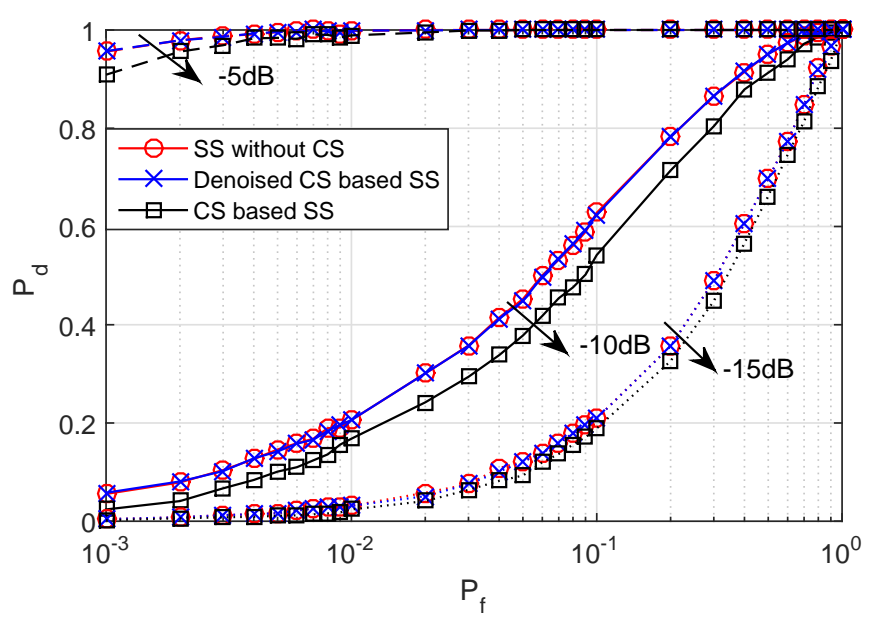

Fig. 5: Proposed denoised compressive spectrum sensing algorithm at single node achieves higher ROC curves than the traditional algorithms with simulated signals, and compression ratio $25 \%$.

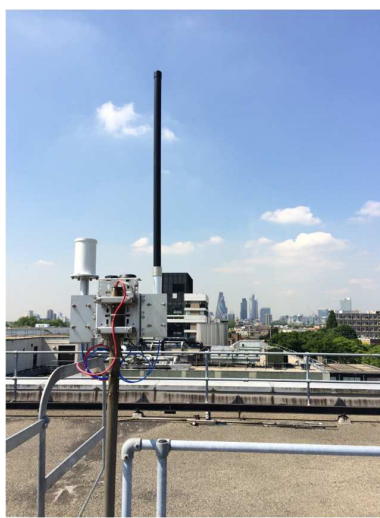

(a). Measurement setup at Queen Mary University of London

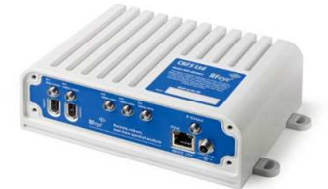

(b). RFeye sensing node

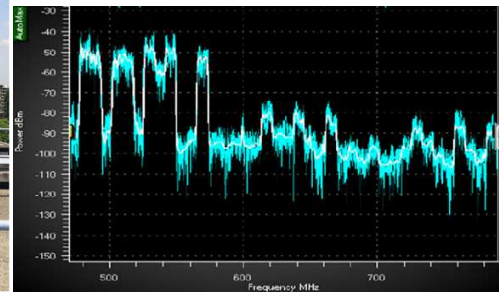

(c). Real-time TVWS signal observed by RFeye sensing node
Fig. 6: Measurement setup for real-time TVWS signals recorded at Queen Mary University of London.

forms the traditional one, which is similar with the results of simulated signals as shown in Fig. 3.

\section{Proposed Two-Phase Cooperative Spectrum Sensing Algorithm Based on LOW-RANK MatriX COMPLETION}

Based on the proposed two-phase single node spectrum sensing algorithm, a new two-phase CSS algorithm based on low-rank $\mathrm{MC}$ is proposed to overcome the deep fading problem. In the first phase, the proposed channel division scheme is extended to the CSS scenario. In the CSS network, each SU is implemented to sense a different $K$-channel group of the $L$ channels to reduce sampling rates, where $K$ channels are sensed by each SU and there are $L$ channels in spectrum of interest. As a result, at least $I\left(I=\frac{L}{K}\right)$ SUs should be implemented to sense the whole spectrum of interest in one sensing period. The positions of active PUs on the whole spectrum of interest are random. Due to deep fading, $J$ SUs are spatially implemented to sense the same $K$-channel

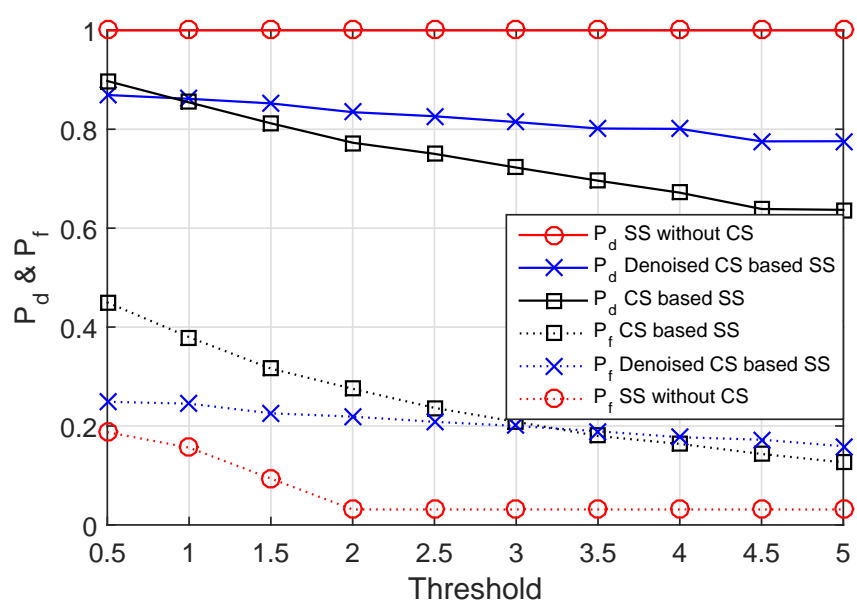

Fig. 7: Detection probability $P_{d}$ and false alarm probability $P_{f}$ comparison of single node spectrum sensing with realtime signals under different thresholds, and compression ratio is $15 \%$.

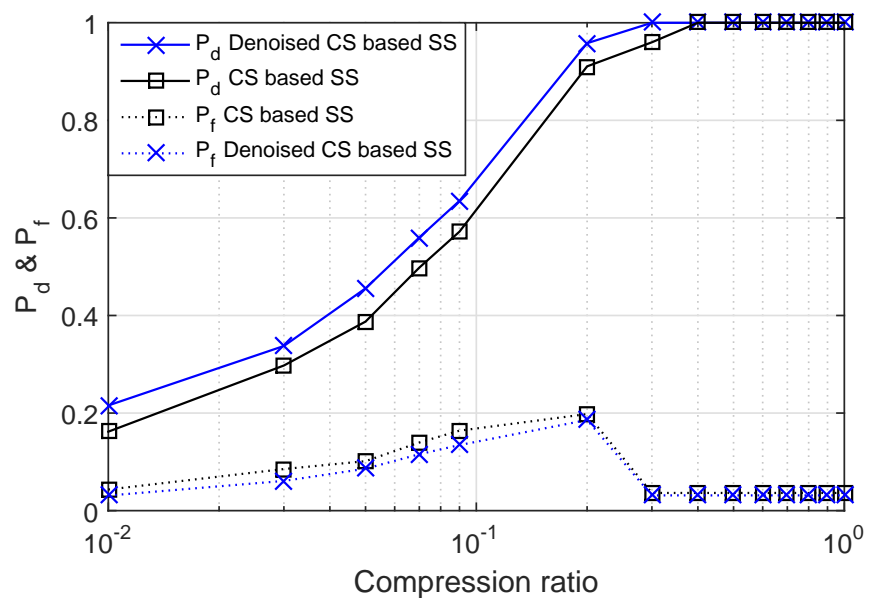

Fig. 8: Proposed denoised compressive spectrum sensing algorithm at single node achieves higher detection probability $P_{d}$ and lower false alarm probability $P_{f}$ than the traditional algorithm with real-time signals under different compression ratios, and threshold is 1.5 .

group. Therefore, the $j$ th $\mathrm{SU}$ implemented to sense the $i$ th $K$-channel group is labeled as $\mathbf{S U}_{i j}$. The whole scenario is shown in Fig. 9. In the second phase, a denoising algorithm is proposed to improve the detection performance of CSS, which is introduced in part B of this section.

\section{A. System model}

Based on the system model in Fig. 9, the CSS algorithm based on low-rank MC can be formulated into a four-step model:

1) Sparse signals received at SUs.

2) Incomplete matrix generation at the FC.

3) $\mathrm{MC}$ at the FC.

4) Decision making at the FC.

1) Sparse signals received at SUs: As noise exists in the transmission channels, signals received at $\mathbf{S U}_{i j}$ is $r_{i j}(t)=$ 


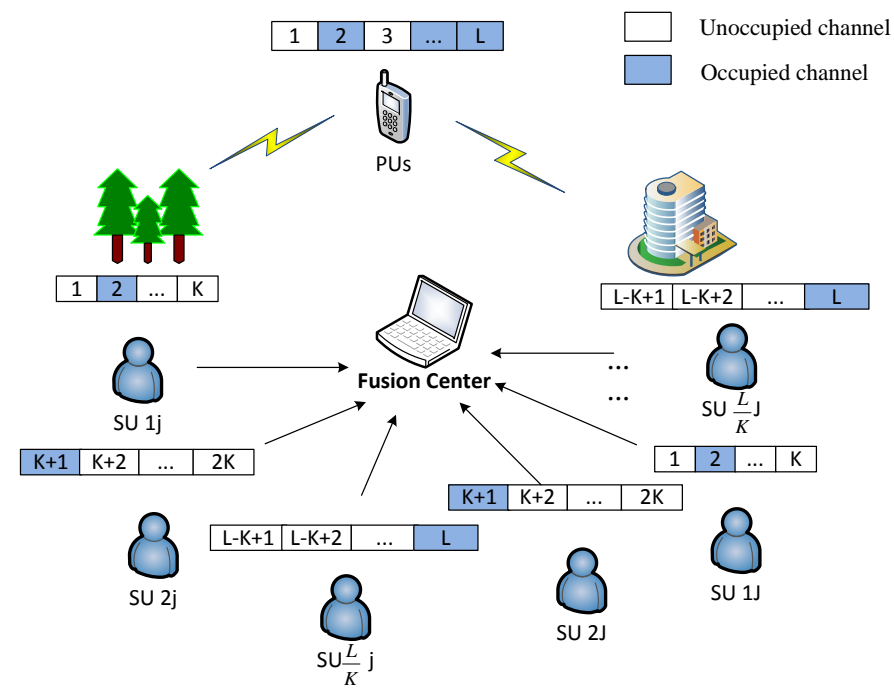

Fig. 9: System model of the proposed channel division scheme in cooperative spectrum sensing algorithm based on low-rank matrix completion.

$h_{i j}(t) * s_{i j}(t)+w_{i j}(t)$, where $s_{i j}(t) \in C^{n \times 1}$ refers to time domain signals of the $i t h K$-channel group received at the $j t h$ receiver $\mathrm{SU}_{i j}, h_{i j}(t)$ refers to the channel gain for the $i$ th $K$ channel group between transmitter and $\mathrm{SU}_{i j}, w_{i j}(t)$ refers to AWGN in the related transmission channels. The frequency domain representations of signals in the $i$ th $K$-channel group which is received by $S U_{i j}$ can be expressed as:

$$
r_{f i j}=h_{f i j} s_{f i j}+w_{f i j},
$$

where $r_{f i j}, h_{f i j}, s_{f i j}$ and $w_{f i j}$ are the DFT of $r_{i j}(t), h_{i j}(t)$, $s_{i j}(t)$ and $w_{i j}(t)$.

At $S U_{i j}$, an AIC sampler $\hat{\Phi}_{i j} \in C^{p \times n}$ is implemented to collect the compressed measurements as follows:

$$
\begin{aligned}
x_{i j} & =\hat{\Phi}_{i j}\left(h_{f i j} F^{-1} s_{f i j}+F^{-1} w_{f i j}\right) \\
& =\hat{\Theta}_{i j}\left(h_{f i j} s_{f i j}+w_{f i j}\right) .
\end{aligned}
$$

2) Incomplete matrix generation at the fusion center: As spectrum utilization is low, the stack of received frequency domain representations $r_{f j}=\sum_{i=1}^{I} r_{f i j}$ are approximately sparse. Each SU only sends $p$ compressed measurements to an FC where $p<n$. At the FC, the matrix $R \in C^{N \times J}(N=I \times n)$ to be recovered shows a low-rank property transformed from the sparse property of signals received at SUs as shown in Fig. 10. In Fig. 10, the circled items refer to the observed measurements as the CS technique is implemented at each SU. This naturally forms a double-sparsity property to use fewer measurements to reconstruct the original signals. In order to avoid poor spectrum resolution in frequency domain and high spectral leakage in each channel, we set the number of the rows $N$ to be equal to the original number of samples for the whole spectrum of interest $I \times n$ rather than the number of channels $I$, which is adopted in [20], [39].

At the FC, only a subset $\Omega \subseteq C^{M \times J}$ of $R$ are collected where $M=I \times p$. We stack all columns $r_{j}$ of $R$ into a long vector as $\operatorname{vec}(R)$. The incomplete matrix $X$ is obtained by:

$$
\operatorname{vec}(X)=\hat{\Theta} \operatorname{vec}(R)=\hat{\Theta} H \operatorname{vec}(S)+\hat{\Theta} H \operatorname{vec}(W),
$$

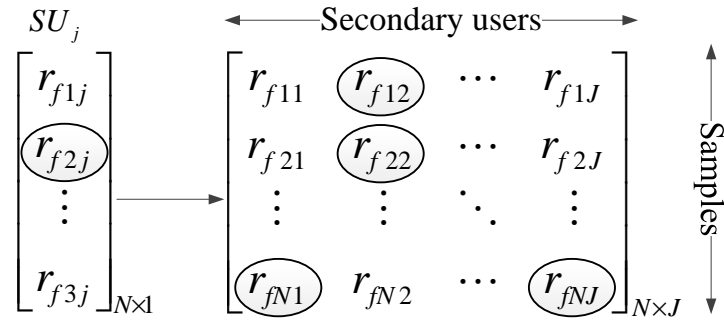

Sparse property Low-rank property

Fig. 10: Matrix to be recovered at the fusion center.

where $h_{f}=\operatorname{diag}\left(\operatorname{diag}\left(h_{f 11}, \ldots, h_{f 1 J}\right), \ldots, \operatorname{diag}\left(h_{f I 1}, \ldots, h_{f I J}\right)\right)$, $H=\operatorname{vec}\left(h_{f}\right)$, and $\hat{\Theta}=\operatorname{diag}\left\{\hat{\Theta}_{11}, \ldots, \hat{\Theta}_{i j}, \ldots \hat{\Theta}_{I J}\right\}$ is the block diagonal matrix. Is it assumed $S$ is the corresponding noiseless matrix of $R$, and $W=R-S$ is the matrix of the corresponding noise contained in $R$. We should recover the unobserved measurements in $S$ from $X$.

3) Matrix completion at the fusion center: The size of the matrix and the computational cost would increase when the number of the rows $N$ is equal to the length of samples $I \times n$ for the whole spectrum of interest to improve the frequency resolution. The signal recovery process is normally performed at SUs in the single node spectrum sensing algorithm, and SUs are normally power limited devices [40]. Therefore, the signal recovery process may cause long delay which will make the final decision invalid for the dynamic spectrum. However, in a CSS network, the MC process can be performed by a powerful device such as the FC to replace the power limited SUs.

With the low-rank property, the complete matrix $R$ can be recovered from a random subset of its items $X$ at the FC. This MC problem is defined as [41]:

$$
\begin{aligned}
& \hat{S}=\arg \min \operatorname{rank}(\hat{S}) \\
& \text { s.t. }\|\hat{\Theta} \cdot H \cdot \operatorname{vec}(\hat{S})-\operatorname{vec}(X)\|_{2}^{2} \leq \hat{\varepsilon}
\end{aligned}
$$

where $\hat{\varepsilon}$ is the upper bound of the noise.

However, (14) is a NP-hard problem [41]. It has been proved that such a NP-hard problem can be converted to the nuclear norm $\|\cdot\|_{*}$ minimization problem as:

$$
\begin{aligned}
& \hat{S}=\arg \min \|\hat{S}\|_{*} \\
& \text { s.t. }\|\hat{\Theta} \cdot H \cdot \operatorname{vec}(\hat{S})-\operatorname{vec}(X)\|_{2}^{2} \leq \hat{\varepsilon}
\end{aligned}
$$

4) Decision making at the fusion center: When the complete matrix $\hat{S}$ is obtained by solving (15), the average energy density of each $K$-channel group can be calculated and compared with the threshold $\lambda_{d}$ defined in (4) to make the final decision. Once the final decision is made, it should be sent back to each SU participating the cooperative networks to help them get access to the vacant channels.

\section{B. The denoised cooperative spectrum sensing algorithm}

Similarly to the denoising algorithm in the proposed twophase single node spectrum sensing algorithm in (6), the $b$ th frequency bin in the recovered signal $\hat{s}_{f i j}$ is compared with the corresponding noise level $\sigma(b)$. If $\hat{s}_{f i j}(b)$ is higher 
than $\sigma(b)$, the measurement observed at the $\mathrm{FC} r_{f i j}(b)$ is kept. Otherwise, the corresponding value is set to be zero to eliminate the influence of noise. This process can be illustrated as:

$$
\hat{s}_{f i j_{-} d}(b)=\left\{\begin{array}{cc}
r_{f i j}(b) & \text { if } \hat{s}_{f i j}(b) \geq \sigma(b) \\
0 & \text { otherwise }
\end{array} .\right.
$$

\section{Computational complexity and performance analyses}

In the low-rank MC based CSS scenario, the computational complexity of solving the MC problem is at the level of $\mathrm{O}\left(N^{3}\right)$, and the $\mathrm{MC}$ is performed at a very powerful FC. As a result, the complexity introduced by MC would not be a key issue to be considered. In such a case, the key issue is the high sampling requirement for wideband spectrum at SUs with limited sensing capability.

In the proposed two-phase CSS algorithm based on lowrank $\mathrm{MC}$, the bandwidth to be sensed at each $\mathrm{SU}$ is reduced to $K$ out of $L$ channels. Additionally, each SU performs subNyqusit sampling and only the collected samples $p$ are sent to the FC which would lower the transmission load in the networks in comparison with the scenario where all the $n$ samples are sent to an FC. Meanwhile, $\frac{L}{K}$ SUs are required to sense the whole spectrum of interest. As the spatial diversity of SUs are utilized to avoid the deep fading problem in CSS network, the more SUs participating in the CSS network, the better detection performance can be achieved. In such a case, each SU only senses part of the spectrum would lead to performance degradation. This tradeoff is illustrated in the following simulations. In large scale M2M communications networks, such kind of performance degradation can be compensated as the number of participating SUs are large.

\section{Numerical Results on Proposed Two-Phase Cooperative Spectrum Sensing Algorithm Based ON LOW-RANK MATRIX COMPLETION}

\section{A. Analyses on simulated signals}

In the multiple node scenario, the spectrum of interest is TVWS with $L=40$ channels. Each SU is assumed to sense a non-overlapping $K$-channel $(K=8)$ group which is the same as the simulation setup of the single node spectrum sensing scenario in section III. The target $P_{f}$ is set to be 0.01 . Transmission channels between the transmitters to the SUs experience frequency-selective fading. In each sensing period, the fading on each channel is time-invariant and it is modeled by setting a random delay and independent Rayleigh fading gains for the multipath fading channels. Without loss of generality, the first SU participating in the cooperative networks is assumed to experience deep fading and the rest of SUs are experiencing Rayleigh fading. In the following simulations, the performance of proposed two-phase CSS algorithm is presented by considering the influence of multipath deep fading, different number of measurements observed at the FC and different network sizes are analyzed.

The detection performance of single node spectrum sensing under deep fading channels in comparison with CSS algorithm under deep fading channels, AWGN channels, and the theoretical curves defined in (10) are shown in Fig. 11. It can be seen that $P_{d}$ of the single node spectrum sensing, which can be considered as the number of SU implemented to sense each $K$-channel group is $J=1$, becomes much lower than the theoretical curves when the transmission channels experience deep fading. As the spatial diversity gain of CSS, the detection performance of CSS algorithm is much improved even though the SUs experiencing deep fading are also in the cooperative network. In the CSS network, the number of SUs being implemented to sense each $K$-channel group is $J=10$. We can see the detection performance of CSS experiencing deep fading is still a bit lower than that of the theoretical curves and the CSS under AWGN channels.

Furthermore, it is noticed that the signal recovery process introduces most of the computational complexities in the fourstep process for the single node spectrum sensing and the CSS algorithms. In the single node spectrum sensing algorithm based on CS, the signal recovery process is performed at the SU. However, in the CSS algorithm based on low-rank $\mathrm{MC}$, the signal recovery process is performed at the FC. SU devices, such as mobile phones and the slave white space devices (WSDs), are normally battery powered [40] or even battery free for those nodes in wireless power transfer model in which the energy is harvested from power beacons. Therefore, the computation complexity should not be too high at SUs. Otherwise, sensing cost would over the sensing capability of SUs and the delay caused by signal recovery would be intolerable. As a result, the spectrum sensing decision may not be meaningful since spectrum occupancy may have changed during the period of signal recovery. However, for the FC, they are normally powerful devices such as base stations and master WSDs. In fact, the size of the to be solved matrix at the FC is much greater than the number of samples to be recovered at SUs in the single node spectrum sensing, and the size of the matrix to be solved at the FC would also influence the performance of the proposed algorithm.

Fig. 12 illustrates the detection performance comparison of the proposed two-phase CSS algorithm based on low-rank MC, low-rank MC based CSS without denoising algorithm, CSS algorithm without CS technique implemented at SUs and the theoretical values as defined in (10) under different number of observed measurements at the FC. In this scenario, the number of SUs being implemented to sense the same $K$ channel group is $J=10$. The number of active PUs in each $K$-channel group is 1 with random position, corresponding to the sparsity level of $12.5 \%$ in the whole spectrum of interest, which is close to the real spectrum occupancy scenario [1], [2]. It is noticed the $P_{d}$ increases when the number of observed measurements at the FC increases from $10 \%$ to $25 \%$. As the $\mathrm{MC}$ error becomes lower with more observed measurements at the FC, the detection performance of proposed denoised MC based CSS algorithm can almost match with that of CSS algorithm without CS implemented at SUs when the observed measurements at the FC is increased to $25 \%$.

Fig. 13 presents the $P_{d}$ of the proposed two-phase CSS algorithm under different network sizes. In this scenario, the number of SUs being implemented to sense the same $K$ channel group is $J=1,5,10,20$ and $J=25$, respectively. In this scenario, the number of observed measurements at the $\mathrm{FC}$ is set to be $25 \%$ of the total measurements. With 


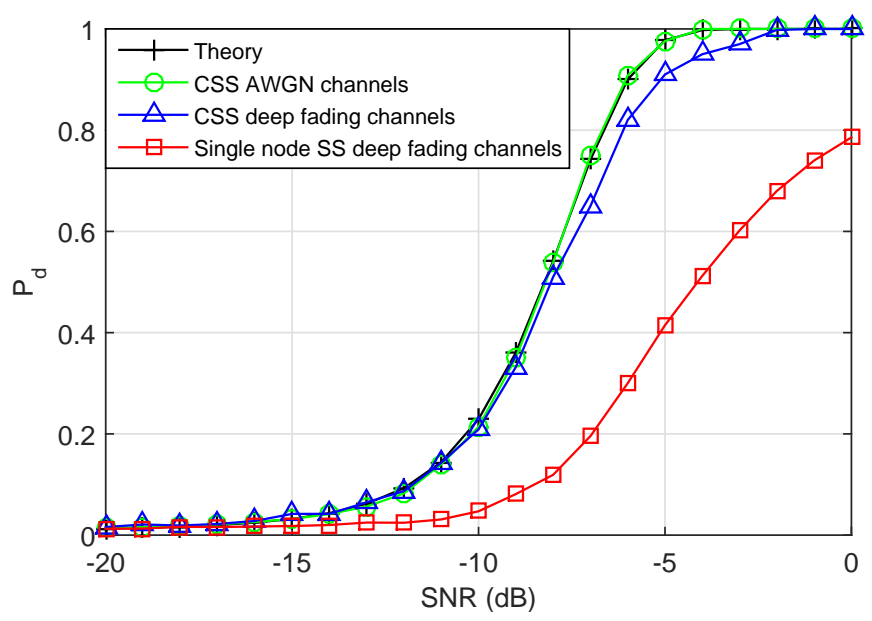

Fig. 11: Probability of detection $P_{d}$ comparison of theoretic curves, CSS under AWGN channels and deep fading channels, and single node spectrum sensing under deep fading channels with simulated signals.

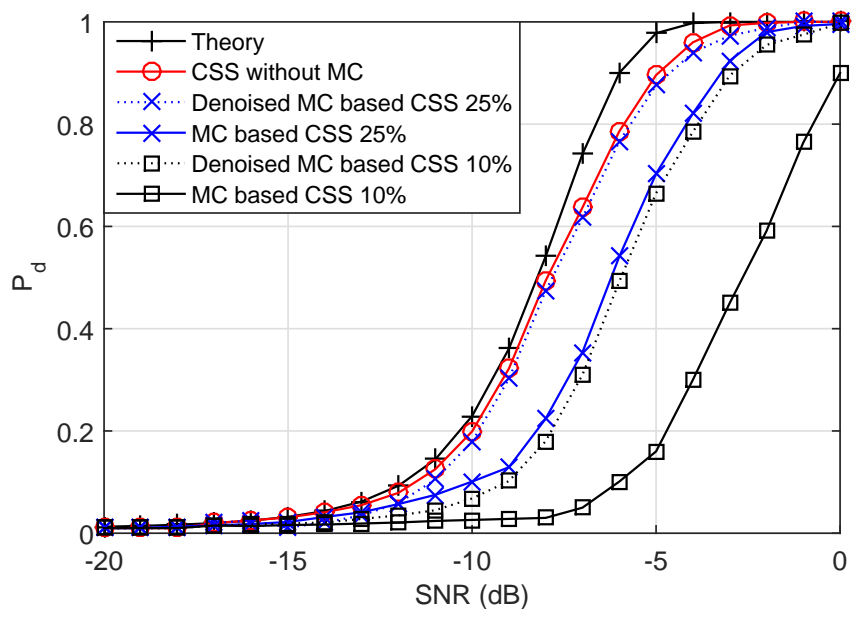

Fig. 12: Probability of detection $P_{d}$ comparison of the proposed two-phase cooperative spectrum sensing algorithm with simulated signals under different number of observed measurements at the fusion center.

decreasing number of SUs participating in the CSS networks, the cooperative gain of CSS networks degrades. The proposed two-phase CSS algorithm is a multiple measurement vector (MMV) model. When the number of SUs implemented to sense the same $K$-channel group is decreased to $J=1$, we obtain a single node spectrum sensing scenario, and the cooperative gain for CSS networks is decreased to zero. In such a case, it becomes a single measurement vector (SMV) model which provides a benchmark for the comparison. We can see that the detection performance increases with increasing number of SUs implemented to sense the same $K$-channel group. It is also noticed that the performance gap for the number of SUs implemented to sense the same $K$-channel group increased from 5 to 10 is higher than that of the number of SUs changing from 10 to 20. As more information about the spectrum is sent to the FC for the final decision making, which refers to more SUs implemented to sense the same
$K$-channel group, the detection performance becomes closer to the theoretical curves. However, when the network size is enlarged, the computational complexity of MC increases. Therefore, it is a balance between the detection performance and the computational complexity of MC. In addition, in the case $J=5$, there are 25 SUs participating in the CSS network as each $\frac{L}{K}=5$ SUs are implemented to sense the whole spectrum of interest at the same location. We can see that the detection performance reaches the theoretic curves with increasing number of SUs. Therefore, the performance degradation caused by the proposed channel division scheme would not be an issue in large scale networks.

\section{B. Analyses on real-time signals}

When the performance of proposed two-phase CSS algorithm based on low-rank MC is verified by the simulated signals, it is further tested on real-time signals collected by the RFeye sensing node installed in our lab as shown in Fig. 6 and a portable RFeye sensing node implemented at different locations in London.

Fig. 14 shows the detection performance comparison of the traditional and the proposed two-phase CSS algorithms under different compression ratios when the real-time signals recorded by the RFeye node are utilized as the signal resources. In this scenario, the number of SUs used to sense the same channels is $J=5$ and the threshold is set to be 1.5 . It is noticed that detection performance of the proposed algorithm would reach the target performance $\left(P_{d}\right.$ is higher than $90 \%$ and $P_{f}$ is lower than $10 \%$ ) when compression ratio is no lower than about $25 \%$. In addition, detection performance of the proposed denoised CSS algorithm is better than the traditional one with increasing compression ratio at the SU, which is the benefit of the proposed denoising algorithm. When compared with Fig 12, we can see that the detection performance becomes better when compression ratio increases. This is also matched with the single node spectrum sensing algorithm based on CS in Fig. 8.

\section{CONCLUSIONS}

In this paper, we proposed two algorithms for wideband spectrum sensing at sub-Nyquist sampling rates to reduce the computational complexity and improve the robustness to channel noise, for the single node and cooperative multiple nodes respectively. The proposed two algorithms were further tested on real-time signals after been validated by the theoretical results and the simulated signals. The analyses results showed that computational complexity of our proposed algorithms is much less than other state-of-the-art methods. The simulation results demonstrated that the detection performance of our proposed spectrum sensing algorithms on both single and multiple nodes are more robust to channel noise than the traditional algorithms.

The proposed two-phase spectrum sensing algorithm based on compressive sensing (CS) at the single node scenario would be a good choice for a fast spectrum sensing, as no data exchange happens during secondary users (SUs) or between SUs and the fusion center (FC). Specifically, if the SU is powerful enough to perform signal recovery, the single node 


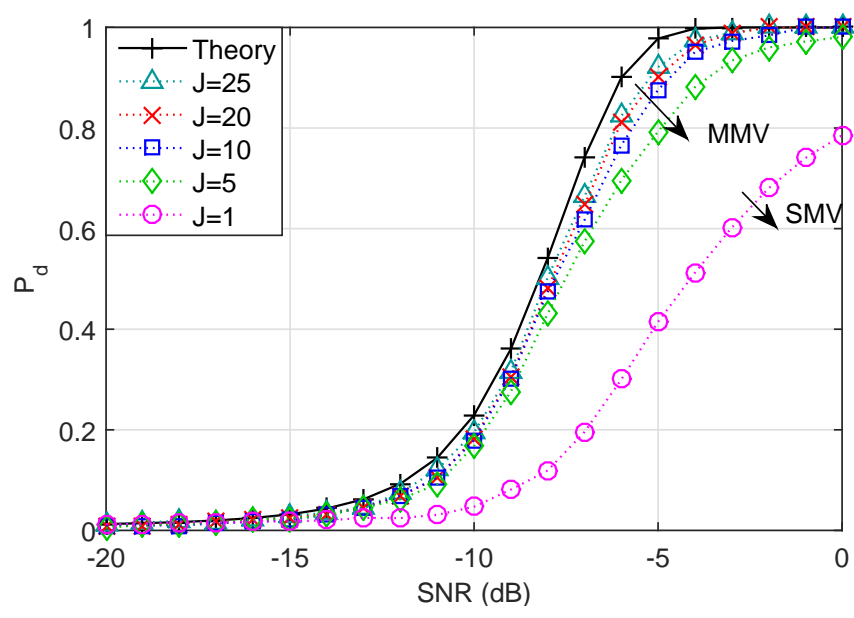

Fig. 13: Probability of detection $P_{d}$ comparison of the proposed two-phase cooperative spectrum sensing algorithm with simulated signals under different network sizes, $25 \%$ measurements observed at the fusion center.

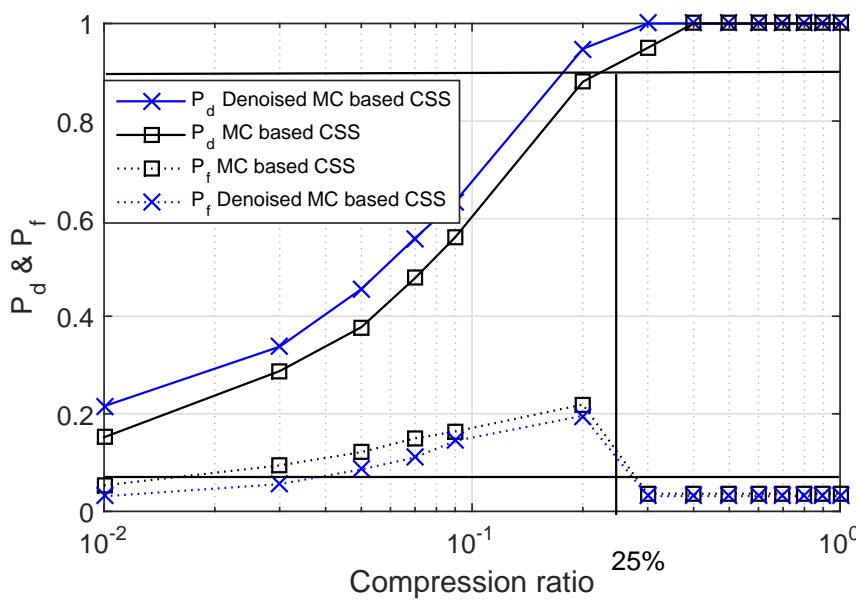

Fig. 14: Proposed two-phase cooperative spectrum sensing algorithm achieves higher detection probability $P_{d}$ and lower false alarm probability $P_{f}$ than the traditional algorithm with real-time signals under different compression ratios, and threshold is 1.5 .

spectrum sensing algorithm based on CS outperforms the cooperative spectrum sensing (CSS) algorithm based on lowrank MC in terms of the network complexity. However, when the transmission environment is poor and SUs are powerlimited or even battery-free devices, the proposed two-phase CSS algorithm would be a better approach to overcome the influence of deep fading by utilizing the spatial diversity of participating SUs, and reduce the power consumption at SUs by performing signal recovery at the FC. Furthermore, it was noticed that CSS would be a typical scenario of dynamic spectrum access promoted by both Federal Communications Commission and the UK Office of Communications, in which master white space devices (WSDs) work as the FC and slave WSDs work as the different participating SUs. Therefore, the proposed two-phase CSS algorithm is a strong candidate for such kind of wideband spectrum sensing scenario in TV while space, particularly for super WiFi, Machine-to-Machine communications, etc.

\section{ACKNOWLEDGMENT}

The authors would like to acknowledge the Engineering and Physical Sciences Research Council (EPSRC) in the UK for their support of this work with Grant No. EP/L024241/1. Mark D. Plumbley was partly supported by a Leadership Fellowship (EP/G007144/1) from the UK EPSRC.

\section{REFERENCES}

[1] P. Kolodzy and I. Avoidance, "Spectrum policy task force," Federal Commun. Comm., Washington, DC, Rep. ET Docket, no. 02-135, 2002.

[2] UK Office of Communications (Ofcom), "Statement on Cognitive Access to Interleaved Spectrum," Jul. 2009. [Online]. Available: http://stakeholders.ofcom.org.uk/binaries/consultations/cognitive/ statement/statement.pdf

[3] I. Mitola, J. and J. Maguire, G.Q., "Cognitive radio: making software radios more personal," IEEE Pers. Commun., vol. 6, no. 4, pp. 13-18, Aug. 1999.

[4] Z. Quan, S. Cui, A. Sayed, and H. Poor, "Optimal multiband joint detection for spectrum sensing in cognitive radio networks," IEEE Trans. Signal Process., vol. 57, no. 3, pp. 1128-1140, Mar. 2009.

[5] Z. Tian and G. B. Giannakis, "A wavelet approach to wideband spectrum sensing for cognitive radios," in Proc. International Conference on Cognitive Radio Oriented Wireless Networks and Communications (CROWNCOM), Mykonos Island, Greece, Jun. 2006, pp. 1-5.

[6] H. Landau, "Necessary density conditions for sampling and interpolation of certain entire functions," Acta Mathematica, vol. 117, no. 1, pp. 37$52,1967$.

[7] E. Candes, "Compressive sampling," in Proc. the International Congress of Mathematicians, vol. 3, Madrid, Spain, Aug. 2006, pp. 1433-1452.

[8] Z. Tian and G. Giannakis, "Compressed sensing for wideband cognitive radios," in Proc. IEEE International Conference on Acoustics, Speech and Signal Processing (ICASSP), Honolulu, HI, Apr. 2007, pp. 13571360.

[9] Z. Tian, Y. Tafesse, and B. M. Sadler, "Cyclic feature detection with subNyquist sampling for wideband spectrum sensing," IEEE J. Sel. Signal Process., vol. 6, no. 1, pp. 58-69, Feb. 2012.

[10] Y. Wang, Z. Tian, and C. Feng, "Sparsity order estimation and its application in compressive spectrum sensing for cognitive radios," IEEE Trans. Wireless Commun., vol. 11, no. 6, pp. 2116-2125, Jun. 2012.

[11] H. Sun, W.-Y. Chiu, and A. Nallanathan, "Adaptive compressive spectrum sensing for wideband cognitive radios," IEEE Commun. Lett., vol. 16, no. 11, pp. 1812-1815, Nov. 2012.

[12] R. Venkataramani and Y. Bresler, "Perfect reconstruction formulas and bounds on aliasing error in sub-Nyquist nonuniform sampling of multiband signals," IEEE Trans. Inf. Theory, vol. 46, no. 6, pp. 21732183, Sep. 2000.

[13] M. A. Lexa, M. E. Davies, J. S. Thompson, and J. Nikolic, "Compressive power spectral density estimation," in Proc. IEEE International Conference on Acoustics, Speech and Signal Processing (ICASSP), Prague, Czech Republic, May 2011, pp. 3884-3887.

[14] D. Ariananda and G. Leus, "Compressive wideband power spectrum estimation," IEEE Trans. Signal Process., vol. 60, no. 9, pp. 4775-4789, Sep. 2012.

[15] D. Cohen and Y. C. Eldar, "Sub-Nyquist sampling for power spectrum sensing in cognitive radios: A unified approach," IEEE Trans. Signal Process., vol. 62, no. 15, pp. 3897-3910, Jul. 2014.

[16] M. Mishali and Y. Eldar, "Blind multiband signal reconstruction: Compressed sensing for analog signals," IEEE Trans. Signal Process., vol. 57 , no. 3, pp. 993-1009, Mar. 2009.

[17] _ - "From theory to practice: Sub-Nyquist sampling of sparse wideband analog signals," IEEE J. Sel. Signal Process., vol. 4, no. 2, pp. 375-391, Apr. 2010.

[18] J. Tropp, J. Laska, M. Duarte, J. Romberg, and R. Baraniuk, "Beyond Nyquist: Efficient sampling of sparse bandlimited signals," IEEE Trans. Inf. Theory, vol. 56, no. 1, pp. 520-544, Jan. 2010.

[19] F. Zeng, C. Li, and Z. Tian, "Distributed compressive spectrum sensing in cooperative multihop cognitive networks," IEEE J. Sel. Signal Process., vol. 5, no. 1, pp. 37-48, Feb. 2011.

[20] Y. Wang, Z. Tian, and C. Feng, "Collecting detection diversity and complexity gains in cooperative spectrum sensing," IEEE Trans. Wireless Commun., vol. 11, no. 8, pp. 2876 -2883, Aug. 2012. 
[21] V. Havary-Nassab, S. Hassan, and S. Valaee, "Compressive detection for wide-band spectrum sensing," in Proc. IEEE International Conference on Acoustics Speech and Signal Processing (ICASSP), Dallas, TX, Mar. 2010, pp. 3094-3097.

[22] J. Treichler, M. Davenport, and R. Baraniuk, "Application of compressive sensing to the design of wideband signal acquisition receivers," US/Australia Joint Work. Defense Apps. of Signal Processing (DASP), Lihue, Hawaii, vol. 5, Sep. 2009.

[23] CRFS, "RFeye Node 20-6," Oct. 2015. [Online]. Available: https: //www.crfs.com/products/nodes/node-20-6/

[24] F. F. Digham, M.-S. Alouini, and M. K. Simon, "On the energy detection of unknown signals over fading channels," IEEE Trans. Commun., vol. 55, no. 1, pp. 21-24, 2007.

[25] I. F. Akyildiz, B. F. Lo, and R. Balakrishnan, "Cooperative spectrum sensing in cognitive radio networks: A survey," Physical Communication, vol. 4, no. 1, pp. 40-62, Mar. 2011.

[26] A. Ghasemi and E. Sousa, "Collaborative spectrum sensing for opportunistic access in fading environments," in Proc. IEEE International Symposium on New Frontiers in Dynamic Spectrum Access Networks (DySPAN), Baltimore, MD, 2005, pp. $131-136$.

[27] Z. Tian, "Compressed wideband sensing in cooperative cognitive radio networks," in Proc. IEEE Global Commun. Conf. (GLOBECOM), 2008, pp. 3756-3760.

[28] J. Bazerque and G. Giannakis, "Distributed spectrum sensing for cognitive radio networks by exploiting sparsity," IEEE Trans. Signal Process., vol. 58, no. 3, pp. 1847-1862, Mar. 2010.

[29] Z. Li, F. R. Yu, and M. Huang, "A distributed consensus-based cooperative spectrum-sensing scheme in cognitive radios," IEEE Trans. Veh. Technol., vol. 59, no. 1, pp. 383-393, Jan. 2010.

[30] W. Zhang, R. Mallik, and K. Letaief, "Optimization of cooperative spectrum sensing with energy detection in cognitive radio networks," IEEE Trans. Wireless Commun., vol. 8, no. 12, pp. 5761-5766, Dec. 2009.

[31] Y. Zeng, Y.-C. Liang, A. T. Hoang, and R. Zhang, "A review on spectrum sensing for cognitive radio: Challenges and solutions," EURASIP J. Adv. Signal Process, vol. 2010, Jan. 2010.

[32] J. Meng, W. Yin, H. Li, E. Houssain, and Z. Han, "Collaborative spectrum sensing from sparse observations using matrix completion for cognitive radio networks," in Proc. IEEE International Conference on Acoustics Speech and Signal Processing (ICASSP), Dallas, TX, Mar. 2010, pp. $3114-3117$.

[33] J. Meng, W. Yin, H. Li, E. Hossain, and Z. Han, "Collaborative spectrum sensing from sparse observations in cognitive radio networks," IEEE $J$. Sel. Areas Commun., vol. 29, no. 2, pp. 327-337, Feb. 2011.

[34] F. Digham, M.-S. Alouini, and M. K. Simon, "On the energy detection of unknown signals over fading channels," in Proc. IEEE International Conference on Communications (ICC), Anchorage, AK, May. 2003, pp. 3575-3579.

[35] Z. Ye, G. Memik, and J. Grosspietsch, "Energy detection using estimated noise variance for spectrum sensing in cognitive radio networks," in Proc. IEEE Wireless Communications and Networking Conference (WCNC), Las Vegas, NV, Mar. 2008, pp. 711-716.

[36] N. Wang, Y. Gao, and X. Zhang, "Adaptive spectrum sensing algorithm under different primary user utilizations," IEEE Commun. Lett., vol. 17, no. 9, pp. 1838-1841, Sep. 2013.

[37] Z. Qin, Y. Gao, M. Plumbley, and C. Parini, "Efficient compressive spectrum sensing algorithm for M2M devices," in Proc. IEEE Global Conference on Signal and Information Processing (GlobalSIP), Atlanta, GA, Dec. 2014, pp. 1170-1174.

[38] "IEEE Standard for Wireless Regional Area Networks Part 22: Cognitive Wireless RAN MAC \& PHY Specifications: Policies and Procedures for Operation in the TV Bands," IEEE Std. 802.22-2011, 2011.

[39] Q. Ling and Z. Tian, "Decentralized support detection of multiple measurement vectors with joint sparsity," in Proc. IEEE International Conference on Acoustics, Speech and Signal Processing (ICASSP), Prague, Czech, May. 2011, pp. 2996-2999.

[40] T. Yucek and H. Arslan, "A survey of spectrum sensing algorithms for cognitive radio applications," IEEE Commun. Surveys Tutorials, vol. 11, no. 1 , pp. 116-130, Mar. 2009.

[41] E. Candes and B. Recht, "Exact matrix completion via convex optimization," Foundations of Computational mathematics, vol. 9, no. 6, pp. 717-772, 2009. 